Marquette University

e-Publications@Marquette

Physics Faculty Research and Publications

Physics, Department of

$1-1-2008$

Fast Domain Wall Motion in Nanostripes with Out-of-Plane Fields

Andrew Kunz

Marquette University, andrew.kunz@marquette.edu

Sarah C. Reiff

Marquette University

Published version. Applied Physics Letters, Vol. 93 (2008). DOI. (C) 2008 American Institute of Physics. Used with permission. 


\title{
Fast domain wall motion in nanostripes with out-of-plane fields
}

\author{
Andrew Kunz ${ }^{\mathrm{a})}$ and Sarah C. Reiff \\ Physics Department, Marquette University, Milwaukee, Wisconsin 53233, USA
}

(Received 4 June 2008; accepted 9 August 2008; published online 26 August 2008)

\begin{abstract}
Controlling domain wall motion is important due to the impact on the viability of proposed nanowire devices. One hurdle is slow domain wall speed when driven by fields greater than the Walker field due to nucleation of vortices in the wall. We present simulation results detailing the dynamics of these vortices including the nucleation and subsequent fast ejection of the vortex core leading to fast domain wall speeds. The ejection is due to the reversal of the core moments by an out-of-plane field. The technique can be used to produce domain walls of known orientation, independent of the initial state. (c) 2008 American Institute of Physics. [DOI: 10.1063/1.2976678]
\end{abstract}

The viability of many proposed nanowire based devices in magnetic recording, sensing, and logic depends on controlling the motion of a domain wall carrying the relevant information. ${ }^{1,2}$ In a thin, narrow nanowire, the magnetization lies in the plane of the wire along the long axis. A magnetic field applied along the long axis of the wire (in-plane) is capable of moving a domain wall located between head-tohead domains. For the proposed applications to be competitive with present devices, the domain wall must be moved quickly and reliably. However, the speed of the domain wall driven by an in-plane field is found to be maximum when the magnitude of the field is equal to the so-called Walker field. ${ }^{3-5}$ When the field is increased beyond the Walker field, the domain wall begins to move in an oscillatory manner due to the nucleation of (anti)vortices inside the domain wall, which must transit the width of the wire before the domain wall begins to move again. ${ }^{6,7}$ The vortices move slowly across the wire leading to low average speeds. It has been shown that it is possible to inhibit the nucleation process by adding roughness to the wire edges or by artificially removing energy from the precessional motion of the magnetic moments in the field. ${ }^{4,8,9}$ A recent publication showed that an underlayer magnetized perpendicularly (out-of-plane) to the plane of the wire leads to fast wall motion for a large range of driving fields. ${ }^{10}$ In this letter, we present simulation results showing that an out-of-plane field is responsible for the fast wall speeds observed. The out-of-plane field does not inhibit the formation of the antivortices, as proposed in Ref. 10, but instead is responsible for reversing the magnetization direction of the nucleated antivortex core. The gyrotropic force then quickly ejects the vortex from where it was nucleated. ${ }^{11}$ Because the antivortex is only in the wire for short periods of time, the average domain wall speed remains high. The outof-plane field makes it easy to control the magnetization of the core, and we present a technique for creating domain walls of a known magnetization direction. Domain wall speed, domain wall nucleation and injection, and pinning and releasing from notches all depend on the relative orientation of the magnetization inside the domain wall. ${ }^{6,12}$

The simulations are carried out by integrating the Landau-Lifshitz (LL) equation of motion in $5 \mu \mathrm{m}$ long wires of variable rectangular cross-sectional area discretized into identical cubes $5 \mathrm{~nm}$ on edge. ${ }^{13,14}$ The material param-

${ }^{a)}$ Electronic mail: andrew.kunz@marquette.edu. eters used for the simulations are consistent with that of Permalloy, and the wall speed is found by finding the time it takes the domain wall to cross the central half of the wire. In Fig. 1, the domain wall speed as a function of the in-plane driving field is presented for a wall moving along a 100 $\times 5 \mathrm{~nm}^{2}$ cross-section wire. When no out-of-plane field is applied, the wall behaves as expected, moving quickly below the critical field and slowly above it. When a large out-ofplane field is applied, the average wall speed is near the critical speed for all in-plane driving fields. The inset of Fig. 1 shows the domain wall velocity as a function of the outof-plane field for three wire thicknesses when driven by a 30 Oe in-plane field. A sharp increase in wall speed is noted at a critical out-of-plane field. As will be explained below, the antivortex nucleates when the magnetization in the domain wall rotates sufficiently out of plane. ${ }^{6}$ Thicker wires have a reduced shape anisotropy, making it easier for the magnetization to rotate out of the plane of the wire. Therefore, as the wire becomes thicker, it is necessary to increase the out-ofplane field to hold the magnetization in plane.

To understand the behavior of the domain wall, it is necessary to review the LL equation of motion for a magnetic moment $m$,

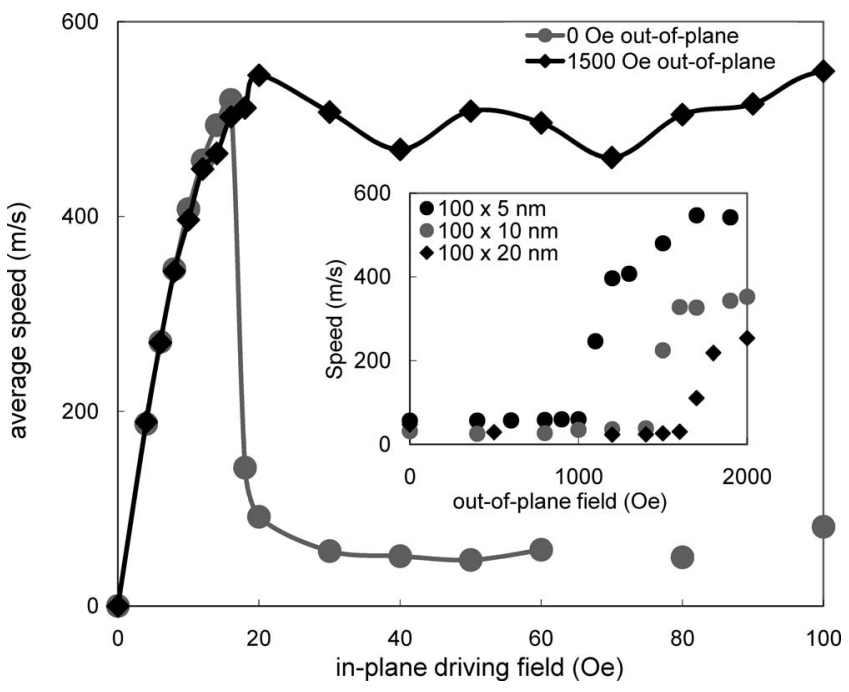

FIG. 1. Domain wall speed in a $100 \times 5 \mathrm{~nm}^{2}$ cross-section wire as a function of longitudinal in-plane driving field with and without an additional out-of-plane applied field. The inset shows the dependence of the critical out-of-plane field on the wire thickness. 


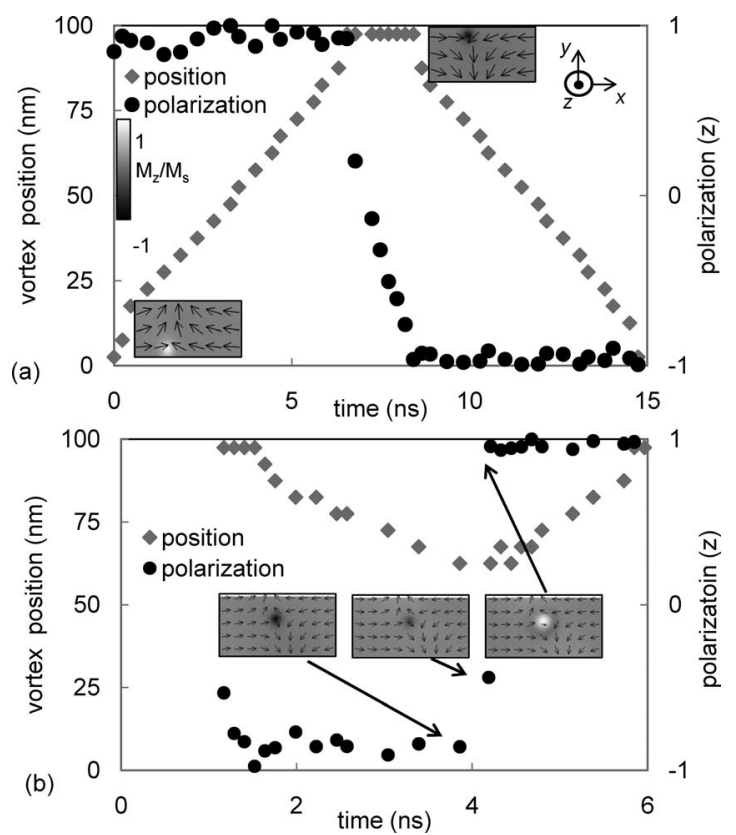

FIG. 2. Plots of the transverse position and polarization of an antivortex core without (a) and with (b) an out-of-plane field in a $100 \mathrm{~nm}$ wide wire. The snapshots show the domain configurations with the grayscale representing the $z$ component of the magnetization. In (b), the out-of-plane field reverses the core leading to the fast ejection of the antivortex.

$$
\frac{\partial \vec{m}}{d t}=-\gamma(\vec{m} \times \vec{H})-\frac{\alpha \gamma}{M_{s}} \vec{m} \times(\vec{m} \times \vec{H}),
$$

where $\gamma$ is the gyromagnetic ratio, $\alpha$ is the phenomenological damping parameter, $M_{s}$ is the saturation magnetization, and $H$ is the total field experienced by the moment. The right hand side of Eq. (1) consists of two primary terms. The first term gives the precessional motion of the magnetic moments and the second is responsible for rotating the magnetization into the direction of the local field. In the presence of a longitudinal in-plane field, the transverse ( $y$-axis) moments of the domain wall are rotated out of the plane of the wire by the first term and the wall is driven along the wire by the second. ${ }^{6}$ The snapshot of the wire in Fig. 2(a) shows that the magnetic moments of the domain wall point in the $+y$ direction. The precessional term of Eq. (1) shows that the driving field rotates the moments into the $-(\hat{y} \times \hat{x})=+\hat{z}$ direction. When the driving field is greater than the Walker field, an antivortex with a core polarization along the $z$-axis is nucleated at the lower edge of the wire with an initial velocity along the $x$-axis. ${ }^{6,15,16}$ The vortex core is then driven by the gyrotropic force $\vec{F}_{g}=\vec{G} \times \vec{v}$ where the gyrovector $G$, has a direction given by the polarization of the vortex core, and $v$ is in the direction of the velocity of the antivortex. ${ }^{17,18}$ In Fig. 2 (a), the gyrotropic force on the nucleated vortex core is initially in the $\hat{z} \times \hat{x}=+\hat{y}$ direction, pushing the vortex into the wire until it moves out the other side, reversing the direction of the transverse magnetic moments ( $y$-component of the magnetization) in the domain wall as it passes. ${ }^{6,7}$ In Fig. 2(a), we plot the transverse position ( $y$-coordinate) of the vortex core and its polarization direction as a function of time in a wire with no out-of-plane field and a 30 Oe inplane driving field. The snapshots show the direction of the magnetic moments near the domain wall and the gray scale shows the $z$-component of the magnetization.

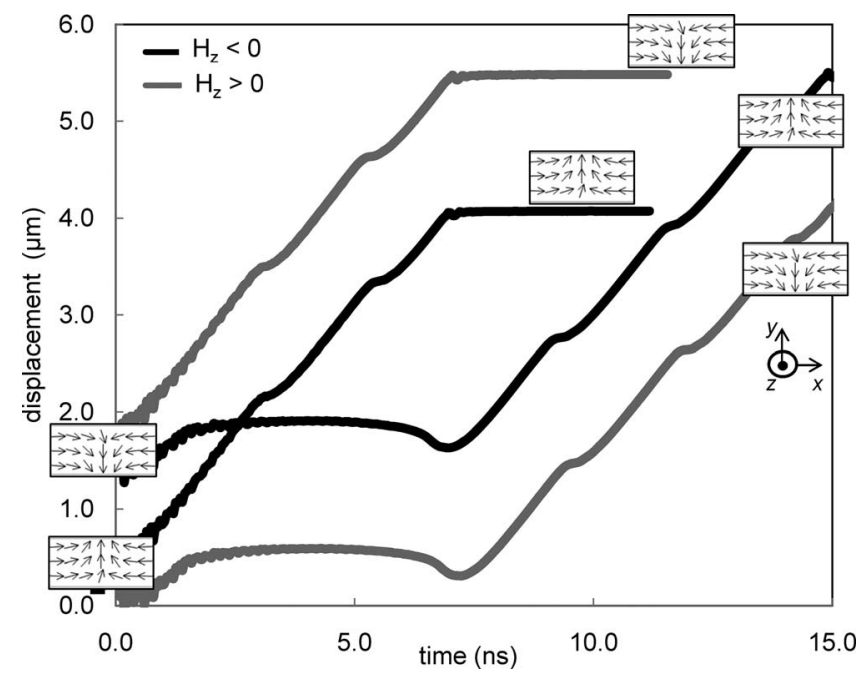

FIG. 3. Wall displacement as a function of time for domain walls of a known initial state for opposite directions of out-of-plane fields in $100 \mathrm{~nm}$ wide wires. The cartoons represent the initial and final orientation of the magnetic moments in the domain wall. A positive out-of-plane field always passes a domain wall oriented down ( $-y$ direction).

In Fig. 2(b), the transverse location of the antivortex core and its polarity is shown as a function of time for a 30 Oe in-plane driving field and an out-of-plane field near the critical field. The three snapshots of the core reversal in Fig. 2(b) are separated by less than $60 \mathrm{ps.} \mathrm{In} \mathrm{this} \mathrm{case,} \mathrm{the} \mathrm{vortex}$ nucleates and moves into the wire before the out-of-plane field reverses the polarization. The damping term in the LL equation of motion is responsible for reversing the direction of the polarization in the antivortex core, leading to a reduction in the Zeeman energy, $E_{\text {Zeeman }}=-\vec{m} \cdot \vec{H}$ as the core moments and out-of-plane field align. This energy is released during the reversal by the formation of spin waves. The reversal takes place via a rotation of the core moments through the plane of the wire due to a direct coupling between the core moments and the large out-of-plane field, not by a vortex/antivortex creation and annihilation process associated with the reversal in nanodisks. ${ }^{19,20}$ Figure 2(b) shows that when the polarization direction changes, the vortex core reverses direction and the vortex is quickly ejected from the wire by the gyrotropic force. Increasing the magnitude of the out-of-plane field decreases the time necessary to reverse the polarization, limiting the amount of time the vortex exists in the wire. The decrease in time recovers the fast domain wall motion.

In Fig. 3, we plot the displacement of a domain wall as a function of time for an initial domain state with the transverse moments in the $+y$ direction and the $-y$ direction under the influence of a large out-of-plane field in a $100 \times 5 \mathrm{~nm}^{2}$ wire. The gyrotropic force depends on the polarization of the nucleated antivortex core. By applying the out-of-plane field in a certain direction, it is possible to create domain walls with a specific transverse direction independent of the starting state. The wall displacements are shifted for ease of viewing. When the transverse wall moments point up $(+y)$, the longitudinal driving field always nucleates an antivortex with a polarization in the $+z$ direction due to the first term in Eq. (1). An out-of-plane field in the $-z$ direction reverses the moments of the core, ejecting the wall quickly so that the transverse domain wall moments always remain in the $+y$ 
direction. However, an out-of-plane field in the $+z$ direction pushes the vortex out the opposite side of the wire, reversing the transverse domain wall moments to the $-y$ direction. Similarly, when started in the $-y$ direction, the wall remains down for a positive out-of-plane field and reverses in a negative field. In summary, an out-of-plane field in the positive direction only passes domain walls oriented down; a negative field passes walls oriented up, as long as the in-plane and out-of-plane fields are both greater than their critical fields. Knowledge of the domain wall orientation is important because it has been shown that domain wall velocity depends on the orientation of the moments. ${ }^{5}$ Similarly, the field needed to inject a domain wall and the ease of pinning and releasing a domain wall from a notch also depends on orientation. $^{12}$

In conclusion, an out-of-plane field applied externally or with appropriate magnetic underlayers can be used to move domain walls at high speed for all driving fields. This represents an order of magnitude improvement in useful driving fields. The out-of-plane field has been shown to reverse the magnetic moments nucleated in the antivortices which allows for fast removal of the vortex via the gyrotropic force. The direction of the external field can be varied to reliably create domain walls of known orientation independent of their initial orientation.

Thanks to Marquette University student Eric Breitbach for writing programs to track the antivortices and Oleg Tchernyshyov for his helpful suggestions. This work was sup- ported by the National Science Foundation, Grant No. DMR0706194 and by a Research Corporation Cottrell College Science Award.

${ }^{1}$ D. A. Allwood, G. Xiong, C. C. Faulkner, D. Atkinson, D. Petit, and R. P. Cowburn, Science 309, 1688 (2005).

${ }^{2}$ S. S. Parkin, M. Hayashi, and L. Thomas, Science 320, 190 (2008).

${ }^{3}$ N. L. Schryer and L. R. Walker, J. Appl. Phys. 45, 5406 (1974).

${ }^{4}$ Y. Nakatani, A. Thiaville, and J. Miltat, Nat. Mater. 2, 521 (2003).

${ }^{5}$ A. Kunz and S. C. Reiff, J. Appl. Phys. 103, $07 D 903$ (2008).

${ }^{6}$ D. G. Porter and M. J. Donahue, J. Appl. Phys. 95, 6729 (2004).

${ }^{7}$ M. Hayashi, L. Thomas, C. Rettner, R. Moriya, and S. S. Parkin, Nat. Phys. 3, 21 (2007).

${ }^{8}$ A. Kunz, IEEE Trans. Magn. 42, 3219 (2006).

${ }^{9}$ J. O. Rantschler, R. D. McMichael, A. Castillo, A. J. Shapiro, W. F. Egelhoff, Jr., B. B. Maranville, D. Pulugurtha, and A. P. Chen, J. Appl. Phys. 101, 033911 (2007).

${ }^{10}$ J.-Y. Lee, K.-S. Lee, and S.-K. Kim, Appl. Phys. Lett. 91, 122513 (2007).

${ }^{11}$ A. A. Thiele, Phys. Rev. Lett. 30, 230 (1973).

${ }^{12}$ D. Atkinson, D. S. Eastwood, and L. K. Bogart, Appl. Phys. Lett. 92, 022510 (2008).

${ }^{13}$ A. Kunz, IEEE Trans. Magn. 43, 2944 (2007).

${ }^{14} \mathrm{LLG}$ Micromagnetic Simulator, http://llgmicro.home.mindspring.com, v2.61, 2007.

${ }^{15}$ O. Tchernyshyov and G.-W. Chern, Phys. Rev. Lett. 95, 197204 (2005).

${ }^{16}$ J.-Y. Lee, K.-S. Lee, S. Choi, K. Y. Guslienko, and S.-K. Kim, Phys. Rev. B 76, 184408 (2007).

${ }^{17}$ O. A. Tretiakov, D. Clarke, G.-W. Chern, Y. A. Bazaliy, and O. Tchernyshyov, Phys. Rev. Lett. 100, 127204 (2008).

${ }^{18}$ R. L. Compton and P. A. Crowell, Phys. Rev. Lett. 97, 137202 (2006).

${ }^{19}$ K.-S. Lee, K. Y. Guslienko, J.-Y. Lee, and S.-K. Kim, Phys. Rev. B 76, 174410 (2007).

${ }^{20}$ R. Hertel, S. Gliga, M. Fähnle, and C. M. Schneider, Phys. Rev. Lett. 98, 117201 (2007). 